\title{
Prospective, double-blind, placebo-controlled trial of low-dose amiodarone in patients with severe heart failure and asymptomatic frequent ventricular ectopy
}

\begin{abstract}
Sudden cardlac death is a common cause of mortality in patients with congestive heart failure. To determine if low-dose amiodarone could reduce sudden death among these patients, a prospective, placebo-controlled, double-blind pllot trial was conducted. One hundred one patients with ejection fractions <30\%, New York Heart Association class III or IV symptoms, and frequent but asymptomatic spontaneous ventricular ectopy (Lown class II to $V$ ) were randomly assigned to treatment with low-dose amiodarone (400 mg/day for 4 weeks and then $200 \mathrm{mg} /$ day) or placebo. Mean follow-up was 357 days (range 4 to 1009 days). Side effects were infrequent and there was no difference in the incidence of side effects between the treatment groups. The frequency of spontaneous ventricular ectopy in the group receiving amiodarone fell from $4992 \pm 1240$ beats/24 hours at baseline to $1135 \pm 494$ beats $/ 24$ hours after 1 month of treatment $(p=0.02)$ and remained low after 6 months, while there was no change in ventricular ectopy among the patients recelving placebo. Despite the reduction in ectopy, there was no improvement in mortality or decrease in the Incidence of sudden death. One-year mortallty by Kaplan-Meier analysis was $28 \%$ in the group receiving amiodarone and $19 \%$ in the group recelving placebo $(p=N S)$. One-year mortallty in patients with $>75 \%$ reduction in ventricular ectopy after 1 month of treatment was $31 \%$ versus $17 \%$ in patlents with $\leq 75 \%$ ectopic suppression ( $p=N S$ ). Although the size of the trial and its statistical power do not eliminate the possibility of a significant reduction in mortality with low-dose amiodarone, any effect is likely to be modest, l.e., <25\%. Therefore low-dose amlodarone can be safely administered to patients with severely impaired myocardial function and will significantly suppress spontaneous ventricular ectopy. However, despite arrhythmia suppression, low-dose amiodarone may not reduce or may have only a modest effect on the incidence of sudden death in patients with heart fallure and asymptomatic ventricular ectopy. (AM HeART J 1991;122:1016.)
\end{abstract}

John M. Nicklas, MD, William J. McKenna, MD, Ralph A. Stewart, MD, Judith K. Mickelson, MD, Sunil K. Das, MD, M. Anthony Schork, PhD, Shirley J. Krikler, MD, Laurie A. Quain, RN, Fred Morady, MD, and Bertram Pitt, MD. Ann Arbor, Mich., and London, England

Sudden cardiac death has been reported to account for nearly half of the mortality among patients with congestive heart failure. ${ }^{1-11}$ Although the pathophysiology is unknown, these sudden deaths are thought to be caused by malignant ventricular arrhythmias. In an attempt to prevent sudden death and improve

From the Divisions of Cardiology, University of Michigan; and the Hammersmith Hospital, London.

Dr. Nicklas was supported by Clinical Investigator Award No. HL01170 from the National Institutes of Health, Bethesda, Md. Dr. McKenna was supported by a grant from the British Heart Foundation.

Received for publication Feb. 5, 1991; accepted April 1, 1991.

Reprint requests: John M. Nicklas, MD, 3910 Taubman, University of Michigan Hospitals, 1500 E. Medical Center Dr., Ann Arbor, MI 48103-0366. 4/1/31124 survival, antiarrhythmic therapy is often prescribed prophylactically. However, the effect of antiarrhythmic agents on the incidence of sudden death and overall mortality in patients with heart failure has not been tested in controlled trials. Therefore we conducted a prospective, double-blind pilot trial comparing treatment with the antiarrhythmic drug, amiodarone, versus placebo in patients with congestive heart failure and frequent asymptomatic ventricular ectopy to determine the safety of the treatment regimen, the feasibility of carrying out a largescale mortality trial with an agent with the potential to cause serious toxicity, and to gain initial insight into the likelihood of reducing the incidence of sudden death with this agent. 


\section{METHODS}

Patients. Patients ages 21 years or older with congestive heart failure referred to the University of Michigan Hospital, the Veterans Administration Hospital in Ann Arbor, or the Hammersmith Hospital in London were eligible for study. The entry criteria were: (1) New York Heart Association (NYHA) class III or IV heart failure symptoms; (2) left ventricular ejection fraction $\leq 30 \%$; and (3) Lown class II to $\mathrm{V}$ ventricular ectopy recorded during 24-hour ambulatory electrocardiographic monitoring. Patients were excluded if they had a history of symptoms attributable to ventricular ectopy including lightheadedness, dizziness, syncope, or if they were already receiving antiarrhythmic drugs. Patients with palpitations were not excluded. All patients gave informed written consent as approved by their hospital's Committee for Human Subjects. The study population consisted of 101 patients, 86 men and 15 women, aged 26 to 78 years (mean 57 years).

Study design. Forty-nine patients were randomly chosen to receive amiodarone and 52 to receive placebo. The dose of amiodarone was $400 \mathrm{mg} /$ day orally for 4 weeks, followed by a maintenance oral dose of $200 \mathrm{mg} / \mathrm{day}$. The study medication was administered in a double-blind fashion. Serial chest roentgenograms, electrocardiograms, and serum potassium and magnesium levels were obtained every 3 months. Chest roentgenograms were examined for evidence of interstitial fibrosis. The development of heart block or intraventricular conduction delay was assessed from the electrocardiograms. Abnormalities in serum electrolyte levels were immediately corrected with oral supplementation. To avoid digitalis toxicity, the dose of digoxin was reduced to $0.125 \mathrm{mg} /$ day in patients receiving $>0.125$ $\mathrm{mg} /$ day of digoxin. Patients were specifically queried for symptoms of photosensitivity, tremor, ataxia, nausea, and constipation every 3 months. Follow-up 24-hour ambulatory monitoring was performed after 1 month and after 6 months of study to assess suppression of ventricular ectopics and complex ventricular ectopy including couplets and ventricular tachycardia. Ventricular tachycardia was defined as three or more consecutive ventricular ectopics at a mean rate $\geq 120$ beats $/ \mathrm{min}$. Side effect and survival data were reviewed by a safety committee (MAS and BP), blinded by group, at 6 -month intervals.

The trial was designed for a mean follow-up of 1 year. Sample size calculations were based on the following assumptions: (1) The 1-year cardiovascular mortality rate in the control population would be $60 \%$. (2) Sudden deaths would account for $50 \%$ of total deaths. (3) Amiodarone would reduce the incidence of sudden death at 1 year by $50 \%$ and improve survival at 1 year by $25 \%$.

Data analysis. Patient deaths were classified as (1) sudden, if the patient deteriorated and died within 1 hour of the onset of symptoms; (2) progressive, if patient deterioration began $>1$ hour prior to death; (3) infarction-related, if death occurred within 1 week of an acute myocardial infarction; or (4) noncardiac. Patients were withdrawn from the study and were considered to have survived sudden death if they experienced syncope caused by documented ventricular tachycardia or if unexplained syncope occurred.
Table I. Baseline clinical characteristics

\begin{tabular}{lccc}
\hline & $\begin{array}{c}\text { Amiodarone } \\
(n=49)\end{array}$ & $\begin{array}{c}\text { Placebo } \\
(n=52)\end{array}$ & $p$ \\
\hline Age (yr) & $56 \pm 1$ & $59 \pm 1$ & 0.18 \\
Male/Female & $41 / 8$ & $45 / 7$ & 0.90 \\
Ischemic/Idiopathic & $25 / 24$ & $28 / 24$ & 0.93 \\
Ejection fraction (\%) & $19 \pm 1$ & $21 \pm 1$ & 0.18 \\
NYHA class III/IV & $39 / 10$ & $44 / 8$ & 0.69 \\
Duration of symptoms (mo) & $32 \pm 6$ & $33 \pm 5$ & 0.82 \\
Medications (\% of patients) & & & \\
$\quad$ Digoxin & 45 & 44 & 0.89 \\
$\quad$ Loop diuretics & 90 & 90 & 0.84 \\
$\quad$ Potassium-sparing diuretics & 38 & 35 & 0.93 \\
$\quad$ Angiotensin-converting & 61 & 65 & 0.82 \\
$\quad$ enzyme inhibitors & & & \\
Serum electrolytes & & & \\
$\quad$ Potassium & $4.4 \pm 0.1$ & $4.5 \pm 0.1$ & 0.47 \\
$\quad$ Magnesium & $2.0 \pm 0.1$ & $2.1 \pm 0.1$ & 0.71 \\
\hline
\end{tabular}

All data were analyzed according to the intentionto-treat principle. Differences in baseline characteristics between treatment groups were analyzed by the Student's $t$ test or by Fisher's exact test. Kaplan-Meier product limit estimates were used to compare the survival of each treatment group. Savage (Mantel-Cox) statistics were used to assess the significance of associations between baseline characteristics and survival.

\section{RESULTS}

Clinical characteristics. There were no significant differences between treatment groups in baseline clinical characteristics (Table I). The average ejection fraction of the study population was $<20 \%$. A greater proportion of patients had NYHA class III $(n=83)$ than NYHA class IV symptoms $(n=18)$. The average frequency of ventricular ectopy was $4900 \pm 830$ beats $/ 24$ hours. Sixty-two percent of the patients had asymptomatic ventricular tachycardia recorded during baseline monitoring. No patient had an abnormal serum potassium or magnesium level at the time of entry into the study. Symptoms of congestive heart failure had been present for $32 \pm 4$ months. Medical therapy included digoxin in $45 \%$, loop diuretics (either furosemide or bumetanide) in $90 \%$, potassium-sparing diuretics (either amiloride, triamterene, or spironolactone) in $36 \%$, and angiotensin-converting enzyme inhibitors in $64 \%$. Baseline characteristics were similar between hospitals except that digoxin was more commonly administered to patients treated at the University and Veterans Hospitals than at the Hammersmith Hospital.

Side effects. Patients were followed for between 4 and 1009 days (mean 357 days). No patient developed interstitial fibrosis or a peripheral neuropathy during the trial. Heart block occurred in one patient receiving amiodarone and appeared to initiate sudden 

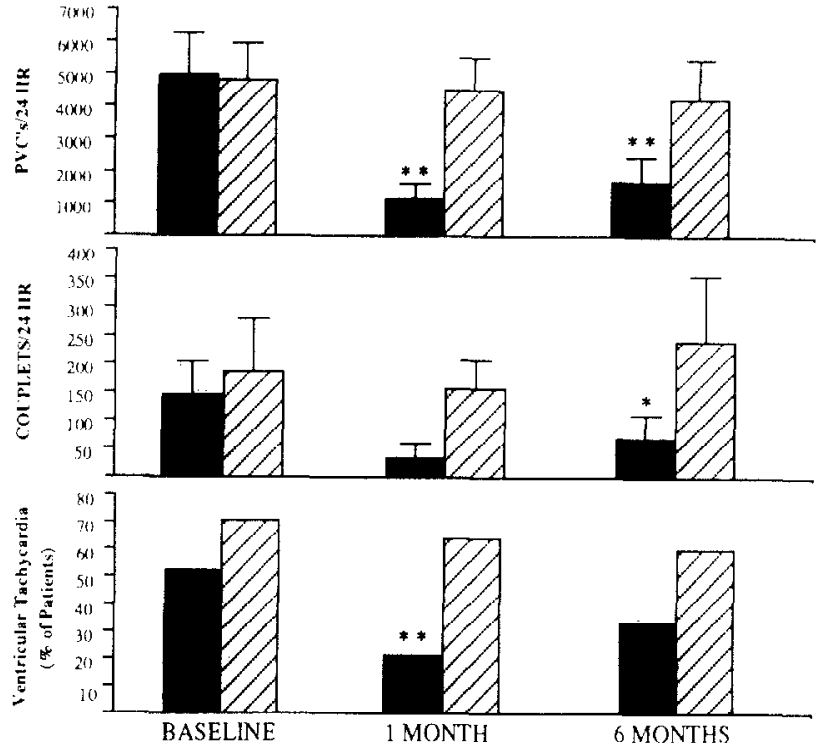

Fig. 1. Relative frequency and complexity of ventricular ectopy in patients receiving amiodarone (solid bars) or placebo (hatched bars) at baseline and after 1 and 6 months of treatment. There were no statistically significant differences between treatment groups at baseline. After 1 month, total premature ventricular contractions ( $P V C$ 's), couplets, and ventricular tachycardia were significantly less frequent in the patients receiving amiodarone compared with those receiving placebo, $p=0.02$, 0.01 , and 0.002 , respectively. Similarly, the frequency of ventricular ectopy decreased from baseline in patients receiving amiodarone but remained unchanged in those receiving placebo $\left({ }^{*} p<0.05 ;{ }^{* *} p<0.02\right)$.

death. The incidence of other side effects was similar in both groups. A total of 12 patients discontinued their study medication, seven in the amiodarone group and five in the placebo group. Reasons for discontinuation among patients receiving amiodarone included: tremor $(n=2)$, non-photosensitive rash $(n=1)$, threefold elevation in hepatic enzymes $(n=1)$, new onset atrial fibrillation $(n=1)$, and relocation to another country $(n=2)$. Reasons for withdrawal among patients receiving placebo included: non-photosensitive rash $(n=1)$, threefold elevation in hepatic enzymes $(n=1)$, new onset atrial fibrillation $(n=1)$, and cardiac transplantation $(n=2)$. Two patients, one from each treatment group, were lost to follow-up.

Ventricular ectopic suppression. Significant suppression of ventricular ectopy in patients treated with amiodarone was observed after 1 month and after 6 months of study (Fig. 1). The frequency of ventricular ectopics in the group receiving amiodarone was reduced from $4992 \pm 1240$ beats/24 hours at baseline to $1135 \pm 494$ beats $/ 24$ hours after 1 month $(p=0.02)$ and to $1686 \pm 770$ beats/24 hours after 6 months $(p=0.14)$. Suppression of more than $75 \%$ of

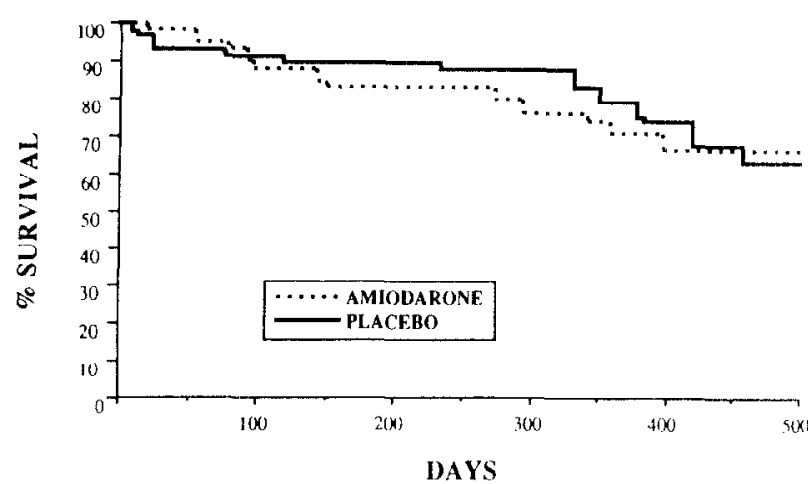

Fig. 2. Kaplan-Meier survival curves for the amiodarone and placebo groups. There was no statistically significant difference between survival in the two groups.

all ventricular ectopics was achieved in $60 \%$ of the patients receiving amiodarone after 1 month and in $75 \%$ after 6 months. In contrast, the frequency of ventricular ectopics did not change significantly in the group receiving placebo, from $4816 \pm 1139$ beats/24 hours at baseline to $4489 \pm 1040$ beats $/ 24$ hours after 1 month $(p=\mathrm{NS})$ and $4213 \pm 1246$ beats $/ 24$ hours after 6 months ( $p=$ NS).

Complex ventricular ectopy was also reduced after 1 month and after 6 months of treatment in the group receiving amiodarone. The frequency of couplets in the group receiving amiodarone was reduced from $145 \pm 59$ couplets/ 24 hours at baseline to $32 \pm 27$ couplets/24 hours after 1 month $(p=\mathrm{NS})$ and to $70 \pm 40$ couplets $/ 24$ hours after 6 months $(p=0.04)$. The frequency of couplets did not change significantly in the group receiving placebo, from $185 \pm 95$ couplets/24 hours at baseline to $156 \pm 51$ couplets/24 hours after 1 month ( $p=$ NS) and to $242 \pm 114$ couplets $/ 24$ hours after 6 months ( $p=$ NS). The proportion of patients with asymptomatic ventricular tachycardia on 24-hour ambulatory monitoring who received amiodarone was $52 \%$ at baseline and $21 \%$ $(p=0.01)$ and $33 \%(p=\mathrm{NS})$ after 1 month and after 6 months of study, respectively. Among patients who received placebo, the proportion with asymptomatic ventricular tachycardia was $71 \%$ at baseline and $64 \%(p=\mathrm{NS})$ and $60 \%(p=\mathrm{NS})$ after 1 and after 6 months, respectively.

Study end points. Twenty-six patients died or survived episodes of sudden death during the trial. All deaths were considered to be cardiovascular and no deaths occurred following infarction. Five deaths were classified as progressive pump failures; two of these patients were receiving amiodarone and three were receiving placebo $(p=N S)$. Twenty-one patients died suddenly; 12 of them were receiving amiodarone and nine were receiving placebo ( $p=$ NS). Five of the 21 patients who experienced sudden death 
ABSOLUTE MORTALITY

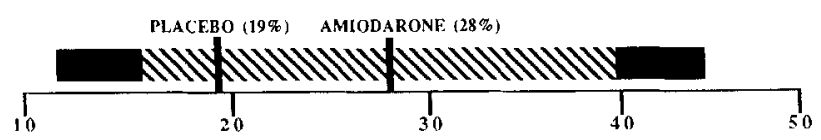

RELATIVE MORTALITY

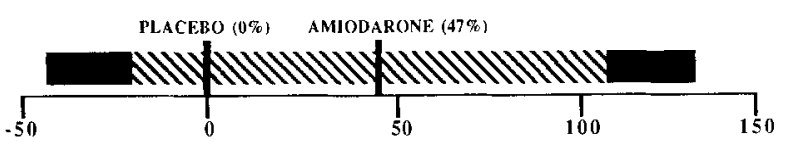

Fig. 3. Absolute and relative mortality in the amiodarone and placebo groups at 1 year. The $80 \%$ (hatched bar) and $90 \%$ (solid bar) confidence limits are shown assuming a 1 year control mortality of $19 \%$.

survived; two who were receiving amiodarone and three who were receiving placebo ( $p=\mathrm{NS}$ ).

Two patients receiving amiodarone died unexpectedly while undergoing ambulatory monitoring. One of these patients died in his sleep with progressive bradycardia over 30 minutes' duration, ending in asystole. The other patient suffered complete heart block without a ventricular escape rhythm. Neither patient had reported previous lightheadedness or syncope and neither had experienced any symptomatic deterioration in exercise tolerance or resting dyspnea.

Survival. There was no difference in the survival rates between treatment groups (Fig. 2). At 1 year, the overall mortality rate was $24 \%-28 \%$ in the group receiving amiodarone and $19 \%$ in the group receiving placebo $(p=\mathrm{NS})$. The absolute and relative differences between groups in 1-year mortality and the statistical power of the trial are illustrated in Fig. 3.

There was also no significant difference in survival between treatment groups in either the ischemic or nonischemic subpopulations (Fig. 4). At 1 year, the mortality rate among patients with ischemic heart disease was $34 \%$ for those receiving amiodarone and $19 \%$ for those receiving placebo $(p=N S)$. The 1 -year mortality among patients with nonischemic cardiomyopathies was $24 \%$ versus $27 \%(p=N S)$ in those receiving amiodarone and placebo, respectively.

Suppression of spontaneous ventricular ectopy was not associated with an improved prognosis. Patients with greater than $75 \%$ suppression of baseline ectopy after 1 month were compared with patients with less suppression (Fig. 5). Four patients died within 1 month of randomization (one receiving amiodarone and three receiving placebo) before undergoing a second 24-hour ambulatory recording to assess the degree of ectopic suppression. At 1 year, the mortal-
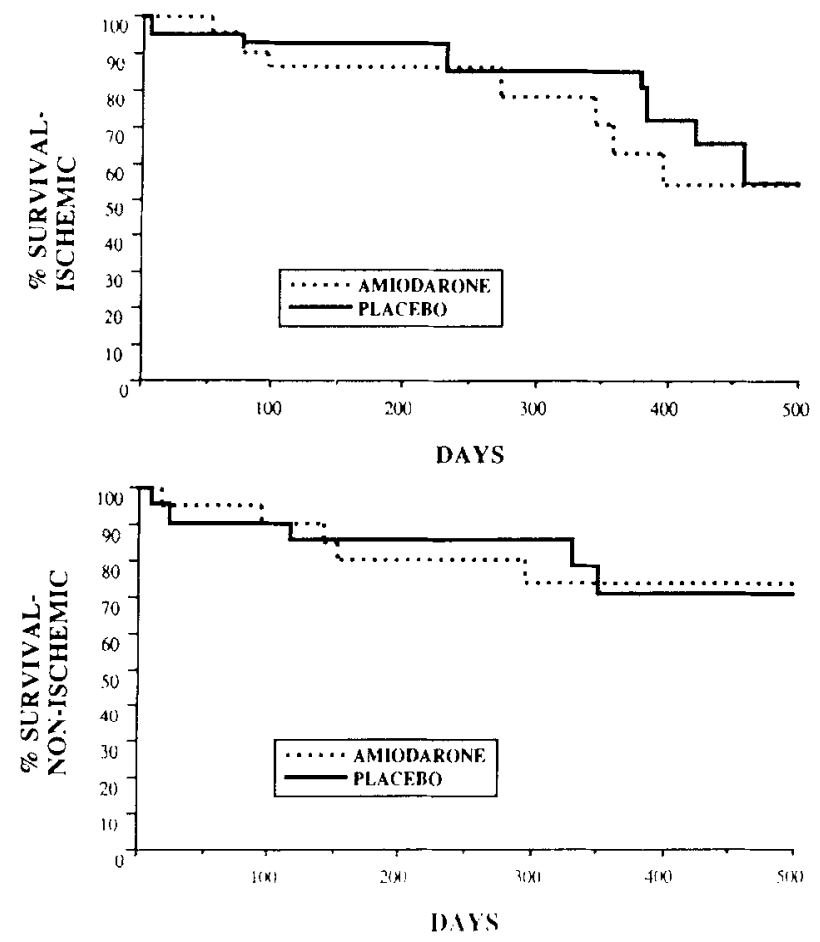

Fig. 4. Kaplan-Meier survival curves for patients receiving amiodarone and placebo subgrouped by etiology. There were no significant differences in survival between treat. ment groups in either the ischemic or nonischemic populations. There was also no significant difference in survival between the ischemic and nonischemic populations.

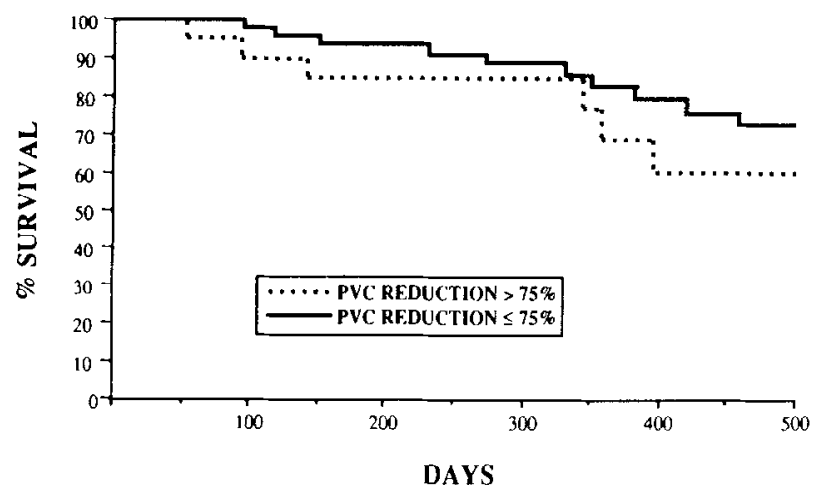

Fig. 5. Kaplan-Meier survival curves for patients whose total ventricular ectopy was markedly suppressed $(>75 \%$ reduction) after 1 month of treatment versus patients whose ectopy was not suppressed ( $\leq 75 \%$ reduction). There was no significant difference in survival between these subgroups based on arrhythmia suppression.

ity rate among patients with $>75 \%$ suppression was $23 \%$ versus $17 \%(p=\mathrm{NS})$ among those with less suppression.

Survival was inversely related to the duration of symptomatic heart failure $(p=0.0065)$ and New York Heart Association functional class $(p=0.033)$. Survival was not related to age, sex, ejection fraction, etiology of the cardiomyopathy, serum levels of 
potassium or magnesium, or treatment with loop diuretics, potassium-sparing diuretics, digoxin, angiotensin-converting enzyme inhibitors, nitrates, or calcium channel blockers.

\section{DISCUSSION}

This study has shown that a low dose of amiodarone can be safely administered to patients with severely impaired left ventricular function and NYHA class III or IV symptoms of congestive heart failure. This dose of amiodarone effectively suppressed ventricular ectopy. However, despite arrhythmia suppression, amiodarone did not reduce the incidence of sudden death or improve survival.

Safety of low-dose amiodarone. The low dose of amiodarone used in this study appeared to be safe for patients with severe heart failure. No patient developed a serious side effect and equal numbers of patients discontinued amiodarone and placebo. Other investigators have also reported that low-dose amiodarone is well tolerated and is not associated with the development of pulmonary toxicity or peripheral neuropathy. ${ }^{12-14}$

However, previous reports of the safety of low-dose amiodarone in heart failure patients suggest that the incidence of some side effects may be dose-dependent. For example, Hamer et al. ${ }^{12}$ reported that lowdose amiodarone $(600 \mathrm{mg} /$ day for 2 weeks followed by $200 \mathrm{mg} /$ day maintenance dosage) improved exercise tolerance in 16 patients with severe left ventricular dysfunction and was safe. Nevertheless, nausea was a significant problem in their study and caused $25 \%$ of the patients to discontinue amiodarone within 6 months. Most of their patients developed nausea during the 2-week loading phase when they were receiving $600 \mathrm{mg} /$ day of amiodarone. In contrast, nausea may have been avoided in our study by using a lower loading dose of the medication $(400 \mathrm{mg} /$ day $)$. Neri et al. ${ }^{13}$ reported that low-dose amiodarone $(600$ $\mathrm{mg} /$ day for 1 week, $400 \mathrm{mg} /$ day for 1 week, followed by a 200 to $400 \mathrm{mg}$ /day maintenance dose) was safely administered to 41 patients with moderately impaired left ventricular function. Although only four patients had to discontinue the medication, $56 \%$ developed adverse effects within 30 months. Six of their patients required pacemaker implantation because of heart block, intraventricular conduction delays, or sick sinus syndrome, and an additional five patients developed sinus bradycardia $(<50$ beats $/ \mathrm{min})$. The absence of these side effects in our study may be attributable to our lower maintenance dose of amiodarone.

Suppression of ventricular ectopy. Although low doses of amiodarone may be safe, they may also be ineffective. However, our study demonstrated that a low dose of amiodarone can significantly suppress both the frequency and complexity of ventricular ectopy in patients with severe heart failure. Total ventricular ectopy was reduced by $75 \%$ in the majority of our patients within 1 month of initiating therapy and in $75 \%$ within 6 months. The incidence of asymptomatic ventricular tachycardia was reduced by $60 \%$ after 1 month and remained low during follow-up. Other investigators have also shown that low-dose amiodarone is effective in reducing ventricular ectopy in heart failure patients. Hamer et al. ${ }^{12}$ reported that ventricular tachycardia was eliminated in 9 of 14 patients after 6 weeks of treatment with low-dose amiodarone. Neri et al. ${ }^{13}$ found that the frequency of ventricular ectopy was suppressed by at least $80 \%$ in 30 of 41 patients after 3 weeks of treatment. Cleland et al. ${ }^{14}$ reported that exercise-induced ventricular tachycardia was suppressed in five of five patients receiving low-dose amiodarone.

Survival. Despite low-dose amiodarone's safety and efficacy in suppressing ventricular ectopy in patients with severe heart failure, we could not detect any reduction in sudden death or prolongation of survival. Although the difference was not statistically significant, overall mortality after 1 year was paradoxically higher in the amiodarone group than in the control group-28\% versus $19 \%$. Similarly, there was no reduction in sudden death among the amiodaronetreated patients compared with the controls. The absence of an effect of amiodarone on survival cannot be attributed to a baseline bias. Randomization of the study population divided the patients into two treatment groups with equivalent characteristics (Table I). The baseline variables that were correlated with mortality-the duration of heart failure symptoms and functional class-did not demonstrate a trend favoring survival in the placebo group.

However, the absence of a beneficial effect of lowdose amiodarone on survival in our study can be attributed to the statistical power of the trial. The sample size calculation had assumed a much higher control mortality than what was observed. Given this low control mortality, reductions in mortality up to $25 \%$ could not have been reliably detected. There are also other explanations for the absence of an improvement in survival with low-dose amiodarone. Although our dose of amiodarone effectively suppressed ventricular ectopy, the degree of suppression was only partial and higher doses of the drug might have been more effective in not only suppressing ectopy but also in preventing sudden death. However, since higher doses of amiodarone appear to be associated with increased toxicity, any reductions in sudden deaths might be offset by increases in other causes of mortality. 
Discrepancy between suppression of ventricular ectopy and prevention of sudden death. The discrepancy between the marked reduction in ventricular ectopy among the amiodarone-treated patients in our study and the absence of any effect on survival is of interest. Both the frequency and complexity of spontaneous ventricular ectopy have been used as indices of the risk of sudden death. ${ }^{1-11}$ This study demonstrates that reductions in asymptomatic ventricular ectopy and the risk of sudden death are not linearly related. A $75 \%$ reduction in ectopy was not associated with a $75 \%$ reduction in the incidence of sudden death. In fact, patients with $>75 \%$ reductions in ventricular ectopy had poorer survival than patients with less suppression (Fig. 4). Investigators in the Cardiac Arrhythmia Suppression Trial (CAST) ${ }^{15}$ found a similar discrepancy between arrhythmia suppression and survival in patients with asymptomatic ventricular ectopy following a myocardial infarction. In their study, patients treated with encainide or flecanide had a three- to fourfold higher incidence of sudden death than patients who received placebo despite effective suppression of ventricular ectopy by both antiarrhythmic agents. Our trial and the CAST study emphasize the need to document the efficacy of antiarrhythmic drugs in reducing sudden deaths rather than in simply suppressing spontaneous ventricular ectopy.

Bradyarrhythmias initiating sudden death. Another explanation for the discrepancy between suppression of ventricular ectopy and ineffective reduction of sudden death in this trial is that not all of the sudden deaths in patients with heart failure are secondary to malignant ventricular tachyarrhythmias. Although patients with congestive heart failure are often found to have frequent and complex ventricular ectopy with episodes of asymptomatic ventricular tachycardia, there is little direct evidence demonstrating that ventricular tachyarrhythmias precipitate sudden death. Most patients die unexpectedly without documentation of the initiating arrhythmia. The largest group of heart failure patients dying suddenly with documentation of the initiating arrhythmia suggests that bradyarrhythmias precipitate sudden death more frequently than tachyarrhythmias. ${ }^{16}$ Thus in this trial amiodarone may have been effective in reducing the frequency of sudden death secondary to ventricular tachyarrhythmias, but because of its effects on nodal and ventricular conduction, it may have increased the incidence of sudden death due to bradyarrhythmias.

Conclusion. Low-dose amiodarone can be safely administered to patients with severe heart failure and it significantly suppresses asymptomatic ven- tricular ectopy. However, despite arrhythmia suppression, low-dose amiodarone does not appear to have a major effect $(>25 \%)$ in reducing the incidence of sudden death. Larger trials will be required to definitively determine the effect of low-dose amiodarone on survival in this population.

The excellent graphics support of Susan J. Schreiber is gratefully acknowledged.

\section{REFERENCES}

1. Franciosa JA, Wilen M, Ziesche S, Cohn JN. Survival in men with severe chronic left ventricular failure due to either coronary heart disease or idiopathic dilated cardiomyopathy. Am J Cardiol 1983;51:831-6.

2. Wilson JR, Schwartz JS, Sutton MSJ, Ferraro N, Horowitz LN, Reichek N, Josephson ME. Prognosis in severe heart failure: relation to hemodynamic measurements and ventricular ectopic activity. J Am Coll Cardiol 1983;2:403-10.

3. Meinertz T, Hofmann T, Kasper W, Treese N, Bechtold H, Stienen U, Pop T, Leitner ERV, Andresen D, Meyer J. Significance of ventricular arrhythmias in idiopathic dilated cardiomyopathy. Am J Cardiol 1984;53:902-7.

4. von Oishausen K, Schäfer A, Mehmel HC, Schwarz F, Senges J, Kübler W. Ventricular arrhythmias in idiopathic dilated cardiomyopathy. Br Heart J 1984;51:195-201.

5. Maskin CS, Siskind SJ, LeJemtel TH. High prevalence of nonsustained ventricular tachycardia in severe congestive heart failure. AM HEART J 1984;107:896-901.

6. Chakko CS, Gheorghiade M. Ventricular arrhythmias in severe heart failure: incidence, significance, and effectiveness of antiarrhythmic therapy. AM HEART J 1985;109:497-504.

7. Holmes J, Kubo SH, Cody RI, Kligfield P. Arrhythmias in ischemic and nonischemic dilated cardiomyopathy: prediction of mortality by ambulatory electrocardiography. Am J Cardiol 1985;55:146-51.

8. Likoff MJ, Chandler SL, Kay HR. Clinical determinants of mortality in chronic congestive heart failure secondary to id. iopathic dilated or to ischemic cardiomyopathy. Am J Cardiol 1987;59:634-8.

9. von Olshausen K, Stienen U, Schwarz F, Kübler W, Meyer J. Long-term prognostic significance of ventricular arrhythmias in idiopathic dilated cardiomyopathy. Am J Cardiol 1988; 61:146-51

10. Hofmann T, Meinertz T, Kasper W, Geibel A, Zehender M, Hohnloser S, Stienen U, Treese N, Just H. Mode of death in idiopathic dilated cardiomyopathy: a multivariate analysis of prognostic determinants. AM HEART J 1988;116:1455-63.

11. Gradman A, Deedwania P, Cody R, Massie B, Packer M, Pitt B, Goldstein S. Predictors of total mortality and sudden death in mild to moderate heart failure. J Am Coll Cardiol 1989; 14:564-70.

12. Hamer AWF, Arkles LB, Johns JA. Beneficial effects of low dose amiodarone in patients with congestive cardiac failure: a placebo-controlled trial. J Am Coll Cardiol 1989;14:1768-74.

13. Neri R, Mestroni L, Salvi A, Pandullo C, Camerini F. Ventricular arrhythmias in dilated cardiomyopathy: efficacy of amiodarone. AM HEART J 1987;113:707-15.

14. Cleland JGF, Dargie HJ, Findlay IN, Wilson JT. Clinical, haemodynamic, and antiarrhythmic effects of long term treatment with amiodarone of patients in heart failure. $\mathrm{Br}$ Heart $J$ 1987:57:436-45.

15. The Cardiac Arrhythmia Suppression Trial (CAST) investigators. Preliminary report: effect of encainide and flecainide on mortality in a randomized trial of arrhythmia suppression after myocardial infarction. N Engl .J Med 1989;321:406-11.

16. Luu M, Stevenson WG, Stevenson LW, Baron K, Walden J. Diverse mechanisms of unexpected cardiac arrest in advanced heart failure. Circulation 1989;80:1675-80. 